\title{
Lapatinib enhances trastuzumab-mediated antibody-dependent cellular cytotoxicity via upregulation of HER2 in malignant mesothelioma cells
}

\author{
RIKI OKITA, KATSUHIKO SHIMIZU, YUJI NOJIMA, TAKURO YUKAWA, \\ AI MAEDA, SHINSUKE SAISHO and MASAO NAKATA \\ Department of General Thoracic Surgery, Kawasaki Medical School, Kurashiki 701-0192, Japan
}

Received July 2, 2015; Accepted August 12, 2015

DOI: $10.3892 /$ or.2015.4314

\begin{abstract}
EGFR/HER2 are frequently expressed in MPM tissues, however, no studies have shown the clinical benefit of using EGFR/HER2-targeting drugs in patients with malignant pleural mesothelioma (MPM). It was reported that the tyrosine kinase inhibitor (TKI) lapatinib enhanced trastuzumab-mediated antibody-dependent cellular cytotoxicity (ADCC) in HER2-positive breast cancer, suggesting that this combination is a promising strategy for MPM treatment. The aim of the present study was to explore the possibility of a TKI combined with trastuzumab to enhance ADCC in MPM cells. Five MPM cell lines were used to test the effects of TKIs targeting EGFR (gefitinib, afatinib and lapatinib) on cell proliferation and the expression of the HER family receptor. The combined effects of TKI with trastuzumab on ADCC were evaluated using the LDH release assay. Additionally, MPM cells were isolated from patients and evaluated for lapatinib-induced upregulation of HER family receptors and trastuzumab- or cetuximab-mediated ADCC. In MPM cell lines, HER2 expression was upregulated by lapatinib, downregulated by afatinib and unaffected by gefitinib. As expected, more trastuzumab bound to MPM cells pretreated with lapatinib than untreated cells, resulting in the enhancement of trastuzumab-mediated ADCC in MPM cells. In patient-derived MPM cells, both HER2 and EGFR were upregulated by lapatinib, resulting
\end{abstract}

Correspondence to: Dr Riki Okita, Department of General Thoracic Surgery, Kawasaki Medical School, Matsushima 577, Kurashiki 701-0192, Japan

E-mail: riki0716okita@yahoo.co.jp

Abbreviations: MPM, malignant pleural mesothelioma; TKI, tyrosine kinase inhibitor; ADCC, antibody-dependent cellular cytotoxicity; DMSO, dimethylsulfoxide; APC, allophycocyanin; PE, phycoerythrin; FITC, fluorescein isothiocyanate; EDTA, ethylenediaminetetraacetic acid; PBS, phosphate-buffered saline; p-, phosphorylated; PBMC, peripheral blood mononuclear cell; NSCLC, non-small cell lung cancer; E:T ratio, effector/target ratio

Key words: malignant pleural mesothelioma, antibody-dependent cellular cytotoxicity, lapatinib, trastuzumab, cetuximab in the enhancement of both trastuzumab- and cetuximabmediated ADCC. Of the three TKIs, only lapatinib enhanced trastuzumab-mediated ADCC via the upregulation of HER2 expression in MPM cells, suggesting that sequential combination of lapatinib and trastuzumab may be a promising strategy for MPM treatment.

\section{Introduction}

Malignant pleural mesothelioma (MPM) is an aggressive thoracic malignancy. Most patients are not eligible for curative surgery due to the advanced stage of the disease (1), and the median survival of MPM with standard chemotherapy (cisplatin combined with pemetrexed) is $\sim 12$ months (2). Despite its aggressiveness, there is currently no established second-line chemotherapy, which makes it difficult to manage this disease. Therefore, new treatment strategies are required.

Clinical samples of MPM cells and cell lines were frequently shown to express the HER family receptors EGFR and HER2 $(3,4)$, which is why clinical studies using EGFR-tyrosine kinase inhibitors (EGFR-TKIs) have been conducted in patients with MPM. However, none of these studies showed any clinical benefits of EGFR-TKIs, requiring other treatment strategies for MPM $(4,5)$. It was recently reported that a sequential combination of lapatinib with trastuzumab enhanced trastuzumab-mediated antibody-dependent cellular cytotoxicity (ADCC) by upregulating HER2 expression in breast (6), esophageal (7) and gastric cancer (8) cell lines. In the present study, we report that the dual EGFR/HER2-TKI lapatinib may upregulate the expression of both EGFR and HER2, while neither the EGFR-TKI gefitinib nor the pan-HER-TKI afatinib demonstrate the same effect. Additionally, we have shown that lapatinib enhanced both trastuzumab- and cetuximab-mediated ADCC in MPM cells isolated from the patient. The sequential combination of lapatinib with trastuzumab and/ or cetuximab may prove to be a promising strategy for MPM patients who have poor treatment options.

\section{Materials and methods}

Cell culture and reagents. The human MPM cell lines NCI-H28, NCI-H2052 and MSTO211 were purchased from the American 
Type Culture Collection (ATCC; Manassas, VA, USA), while the MESO1 (9) and MESO4 (9) cell lines were purchased from RIKEN BRC through the National Bio-Resource Project of the MEXT (Tsukuba, Japan). The genotypes of all cell lines were identified with the GenePrint 10 STR system (Promega, Madison, WI, USA). All the cell lines were maintained in culture in RPMI-1640 medium with $2 \mathrm{mM}$ L-glutamine supplemented with $10 \%$ FBS (both from Invitrogen, Carlsbad, CA, USA) and $50 \mathrm{U} / \mathrm{ml}$ penicillin streptomycin (Sigma-Aldrich, St. Louis, MO, USA) at $37^{\circ} \mathrm{C}$ in a humidified atmosphere with $5 \% \mathrm{CO}_{2}$. For cell culturing, gefitinib (Cayman Chemical, Ann Arbor, MI, USA), afatinib and lapatinib (both from Selleckchem, Houston, TX, USA) were dissolved in dimethylsulfoxide (DMSO) (Sigma-Aldrich). Rituximab, trastuzumab (both from Roche, Basel, Switzerland) and cetuximab (Merck Serono, Darmstadt, Germany) were dissolved in saline. Allophycocyanin (APC)-labeled EGFR (G46-2.6), APC-labeled HER2 and phycoerythrin (PE)-labeled HER3 as well as APC- and PE-labeled anti-mouse IgG1к (MOPC-21)

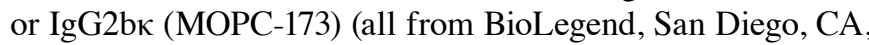
USA) were used for flow cytometry. Fluorescein (FITC)conjugated trastuzumab and FITC-conjugated rituximab were kindly provided by Professor Rolf Kiessling (Karolinska Institutet, Sweden). Cetuximab was labeled with FITC using SureLINK FITC Labeling kit (KPL Inc., Gaithersburg, MD, USA) according to the manufacturer's protocol.

Isolation of MPM cells from patients. The present study was approved by the Institutional Review Board of the Kawasaki Medical School (no. 1433-3). After written informed consent was given, tissue samples or malignant effusions were collected from patients with MPM. Tumor tissue was minced into pieces with ethylenediaminetetraacetic acid (EDTA; Invitrogen) before the centrifugation. The MPM cells were isolated through density centrifugation and were incubated. The MPM cells were used for further experiments after the cells were grown and reached high enough numbers.

WST cell proliferation assay. MPM cells were cultured in triplicate wells of 96-well flat-bottomed plates (Asahi Glass, Tokyo, Japan) with 0.01-10 $\mu \mathrm{M}$ afatinib, gefitinib or lapatinib in $100 \mu \mathrm{l}$ of culture medium for $48 \mathrm{~h}$, then $10 \mu \mathrm{l}$ of WST regent (Roche) were added to wells for $4 \mathrm{~h}$ according to the manufacturer's protocol. Colorimetric reaction was measured by a spectral scanning Varioskan Flash multimode reader (Thermo Scientific, Waltham, MA, USA). TKI-mediated inhibition of cell proliferation was calculated with following formula: $100 \mathrm{x}$ (absorbance of the wells treated with TKI/absorbance of wells treated with DMSO).

Analysis of cell surface molecules by flow cytometry. Tumor cells were washed in phosphate buffered solution (PBS)(-) (Invitrogen), were detached using EDTA (Sigma-Aldrich) and were analyzed for cell surface expression of EGFR, HER2 and HER3 by direct immunofluorescence. Stained cells were acquired on a FACSCanto II (BD Biosciences, Franklin Lakes, NJ, USA) and analyzed using FlowJo software 6.4.7 (Tree Star, Ashland, OR, USA). Both FITC-conjugated trastuzumab and cetuximab were used to evaluate the expression of the trastuzumab-binding site and the cetuximab-binding site, respectively, on the MPM cells, while FITC-conjugated rituximab was used as an isotype control.

Western blot analysis. Cells were washed in PBS(-), lysed over 15 min with CelLytic (Sigma-Aldrich) lysis buffer with a protease inhibitor (protease inhibitor cocktail) and a phosphatase inhibitor (phosphatase inhibitor cocktail 2) (both from Sigma-Aldrich). Cell debris was removed at $12,000 \mathrm{x} \mathrm{g}$ for $15 \mathrm{~min}$. The supernatants were collected and the protein concentrations were analyzed using a BCA protein assay (Takara Bio, Otsu, Japan) according to the manufacturer's protocol. Equal amounts of protein were separated on 4-12\% NuPAGE Bis-Tris acrylamide gels with MES running buffer and transferred to polyvinylidene difluoride membranes (all from Life Technologies, Carlsbad, CA, USA). Blots were blocked for $30 \mathrm{~min}$ in PBS(-) with $0.05 \%$ of Tween-20 (SigmaAldrich) buffer (PBS-T) and 2\% non-fat dry milk, followed by incubation for 2 days at $4^{\circ} \mathrm{C}$ in PBS-T with $2 \%$ non-fat dry milk and primary antibody against: EGFR, phosphorylated EGFR (Tyr1068) (pEGFR), HER2, phosphorylated HER2 (Tyr1221/1222) (pHER2) or $\beta$-actin (Cell Signaling Technology, Beverly, MA, USA). After two washes in PBS-T, membranes were incubated with HRP-linked goat anti-rabbit or anti-mouse $\mathrm{IgG}$ antibodies (Cell Signaling Technology) for $1 \mathrm{~h}$ at room temperature. Blots were visualized by enhanced chemiluminescence with an ECL prime system (GE Healthcare, Fairfield, CT, USA), and images were captured using a LAS-4000 camera system (Fujifilm, Tokyo, Japan). Membranes were stripped in stripping buffer (Takara Bio) for $30 \mathrm{~min}$ at room temperature and reprobed up to two times.

ADCC assay. The present study was approved by the Institutional Review Board of the Kawasaki Medical School (no. 1433-3). Peripheral blood mononuclear cells (PBMCs) were collected from healthy donors using density centrifugation, incubated overnight with $100 \mathrm{IU} / \mathrm{ml}$ of human recombinant IL-2 (Teceleukin, Shionogi, Osaka, Japan) and used as effector cells. MPM cell lines or MPM cells from patients were cultured into a 6-well plate and treated with DMSO or $1 \mu \mathrm{M}$ of afatinib or lapatinib for $24 \mathrm{~h}$, for subsequent use as target cells. The ADCC was evaluated using an LDH release assay (CytoTox 96 Non-Radioactive Cytotoxicity Assay; Promega) in 96-well U-bottom plate (BD Biosciences) with $100 \mathrm{ng} / \mathrm{ml}$ of trastuzumab, cetuximab or rituximab as irrelevant control, according to the manufacturer's protocol. After $4 \mathrm{~h}$ co-incubation, LDH release in the supernatants was measured by Varioskan Flash multimode reader. The percentage of specific lysis was calculated: $100 \mathrm{x}$ (experimental release -spontaneous release)/(maximum release - spontaneous release).

Statistical analysis. Differences in means were evaluated with two-way ANOVA multiple comparisons, with a Bonferroni post test. All analyses were performed using GraphPad Prism 5 software (GraphPad Software Inc., La Jolla, CA, USA) at a significance level of $5 \%(\mathrm{p}<0.05)$.

\section{Results}

Both EGFR and HER2 are widely expressed, while HER3 is not expressed on MPM cells. MPM tissue was collected from 


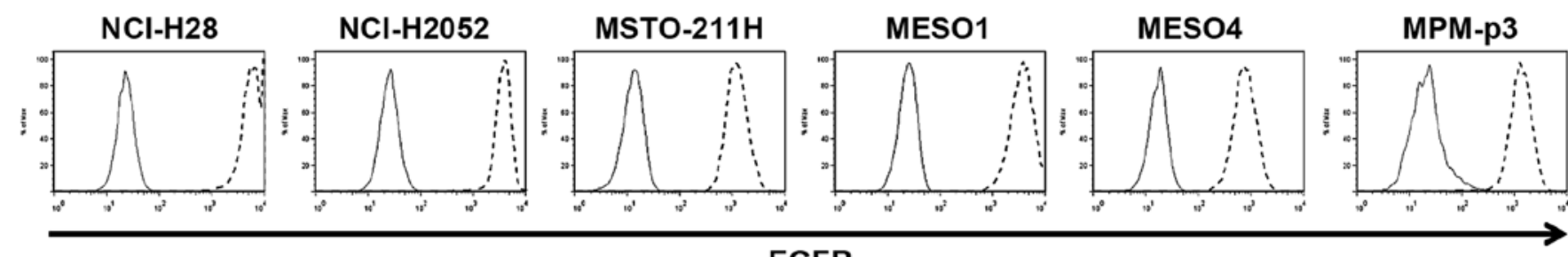

EGFR

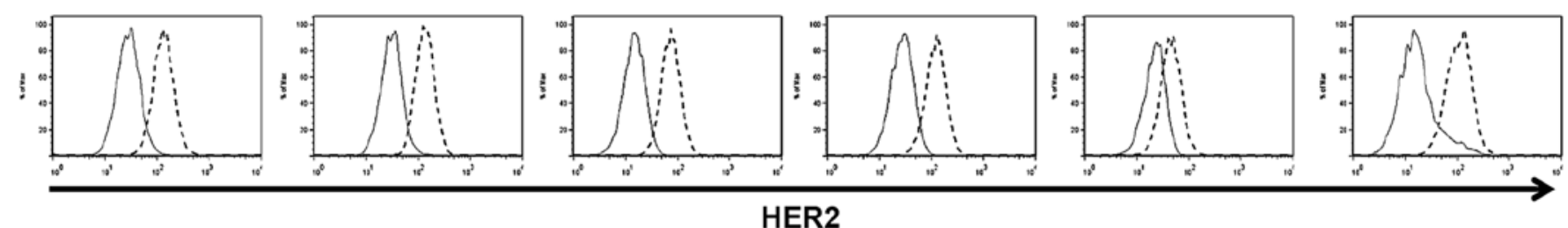

HER2
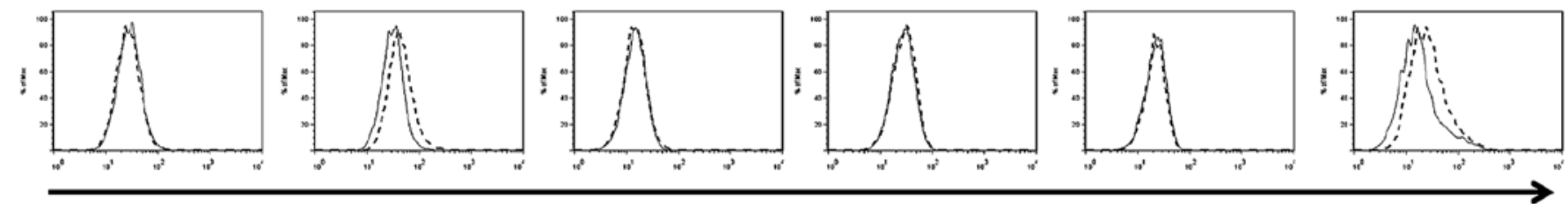

HER3

\section{: Isotype ctr : : specific mAb}

Figure 1. The expression of EGFR, HER2 and HER3 in malignant pleural mesothelioma cells. The basal expression of each cell surface molecule was assessed by flow cytometry. The cells were stained with an isotype control (solid line) or a specific antibody for the indicated molecule (dashed line).

three patients, however, we failed to acquire enough viable MPM cell samples from all tissue samples. In contrast, we collected MPM cells from malignant effusion which could be used for following experiments. It is well known that both EGFR and HER2 are widely expressed in MPM tissue $(3,4)$. First, the basal expressions of EGFR, HER2 and HER3 were determined in five MPM cell lines and the MPM cells from a patient (MPM-p3) using flow cytometry. The EGFR was commonly highly expressed in all MPM cells, while HER2 was also expressed in all MPM cells. In contrast, the expression of HER3 was rare and its expression level was quite low (Fig. 1).

Lapatinib and afatinib strongly inhibit the cell proliferation of MPM cell lines, but gefitinib does not. The first-generation EGFR-TKI, gefitinib, is a powerful tool for EGFR driver mutation-positive non-small cell lung cancer (NSCLC) but invalid for NSCLC with an EGFR gatekeeper mutation such as T790M (10), while a second generation pan-HER inhibitor afatinib overcame T790M in NSCLC cells (11). Lapatinib is a dual EGFR/HER2-TKI with clinical benefit for HER2 overexpressing breast cancer (12) but resistance to T790M (13). Five MPM cell lines, as well as MPM-p3 cells, were treated with various concentrations of TKIs. Both afatinib and lapatinib clearly inhibited cell proliferation of MPM cells, while gefitinib had a slight effect on cell proliferation of MPM cells (Fig. 2).

Lapatinib upregulates HER2 expression while afatinib downregulates HER2 expression in MPM cells. We assessed whether each TKI affected on the expression of EGFR and HER2 in MPM cells (Fig. 3). Tumor cells were treated with a TKI for $24 \mathrm{~h}$, then the expression of EGFR and HER2 was assessed by flow cytometry. Notably, afatinib downregulated the expression of EGFR in NCI-H28 and NCI-H2052 cells and also downregulated the expression of HER 2 in all cell lines, while lapatinib clearly upregulated HER2 expression in all the cell lines. In MPM-p3 cells, afatinib upregulated EGFR and downregulated HER2, while lapatinib upregulated expression of both EGFR and HER2. Gefitinib had no effect on the expression of EGFR and HER2 in the cell lines, or in the MPM-p3 cells.

TKIs have different effect on inhibition of EGFR/HER2. To investigate why the effects on the expression of HER2 were different between TKIs, MPM cells were treated with $1 \mu 1$ of each TKI for $1 \mathrm{~h}$, then both EGFR and HER2 signaling were assessed by western blot analysis in NCI-H28 and NCI-H2052 cells. Gefitinib weakly inhibited the phosphorylation of both EGFR and HER2. Notably, both afatinib and lapatinib strongly and similarly inhibited the phosphorylation of EGFR and HER2 in both cell lines (Fig. 4).

Lapatinib enhances trastuzumab binding with MPM cells, resulting in enhanced trastuzumab-mediated ADCC in MPM cell lines. To evaluate whether lapatinib enhances trastuzumab binding with tumor cells, we used FITC-conjugated trastuzumab in two MPM cell lines (NCI-H28 and NCI-H2052) in which lapatinib clearly upregulated HER2 expression for flow cytometry. In line with our FACS data in Fig. 3, lapatinib upregulated the trastuzumab binding site in both NCI-H28 and NCI-H2052 cells (Fig. 5A and B). Next, trastuzumab-mediated ADCC was evaluated. Lapatinib enhanced trastuzumab-mediated ADCC compared with target cells treated with DMSO in both cell lines, while afatinib did not enhance trastuzumab mediated-ADCC (Fig. 5C and D). 

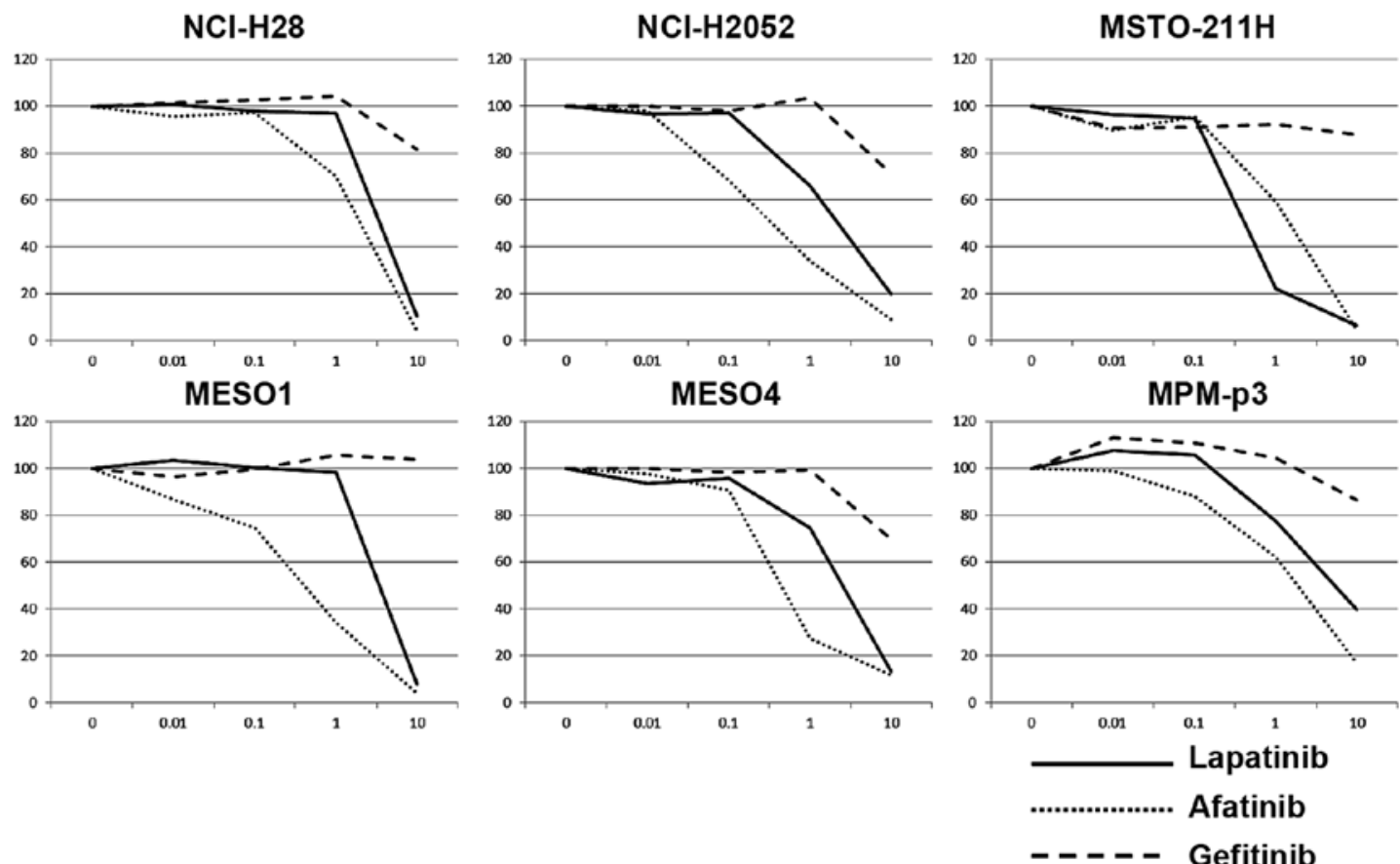

Figure 2. Tyrosine kinase inhibitors inhibited cell proliferation in malignant pleural mesothelioma cells. Five malignant pleural mesothelioma (MPM) cell lines and tumor cells from a patient with MPM were treated with DMSO or indicated concentrations of each tyrosine kinase inhibitor (TKI) for $48 \mathrm{~h}$. After incubation, WST cell proliferation assays were performed. Representative data of three independent experiments (MPM cell lines) and a single experiment (patient-derived MPM cells) are shown.

$\mathrm{NCl}-\mathrm{H} 28$

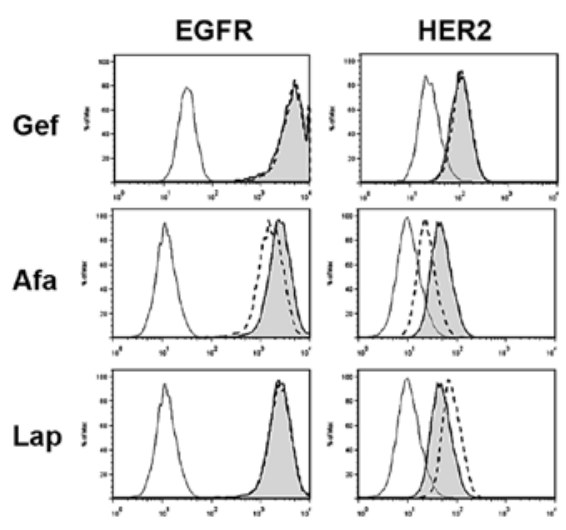

MESO1

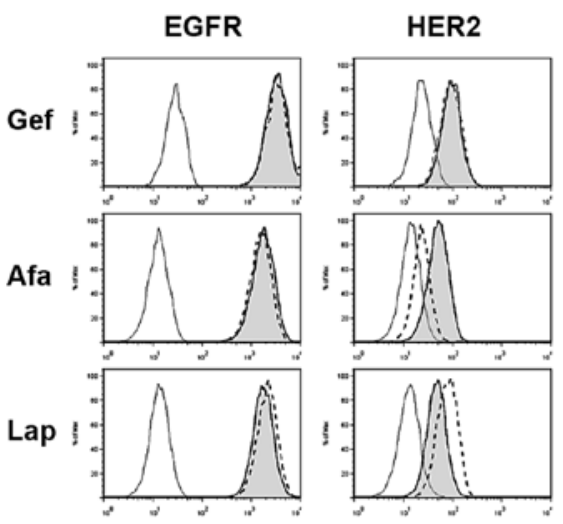

$\mathrm{NCl}-\mathrm{H} 2052$

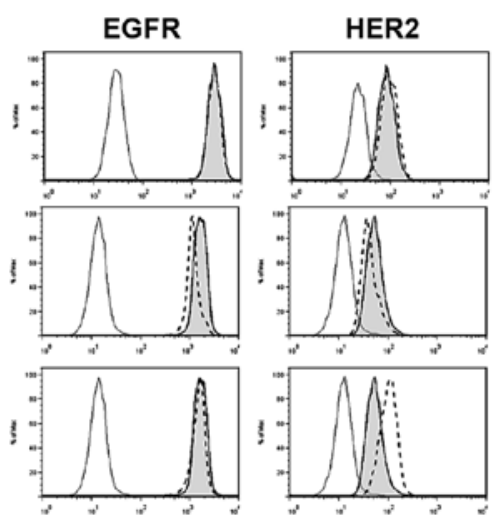

MESO4

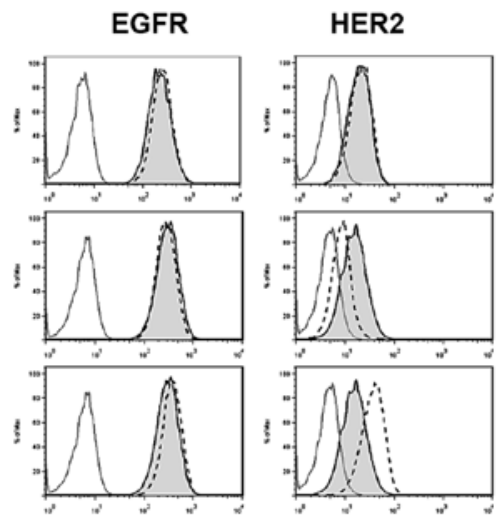

$\bigwedge:$ Isotype ctr $\bigcap$ :
MSTO-211H

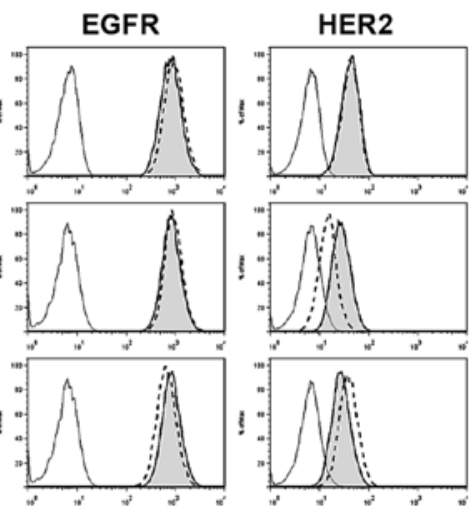

MPM-p3

EGFR

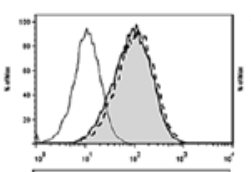

HER2

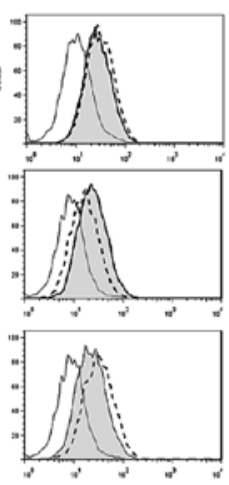

DMSO $1 \mu$ M of EGFR-TKI

Figure 3. The effect of tyrosine kinase inhibitors on the expression of EGFR, HER2 and HER3 in malignant pleural mesothelioma cells. Five malignant pleural mesothelioma (MPM) cell lines and a patient-derived MPM cells were treated with DMSO or $1 \mu \mathrm{M}$ of each tyrosine kinase inhibitor (TKI) for $24 \mathrm{~h}$, then the expression of EGFR, HER2 and HER3 on tumor cells were assessed by flow cytometry. Representative data of three independent experiments (MPM cell lines) and a single experiment (patient-derived MPM cells) are shown. 


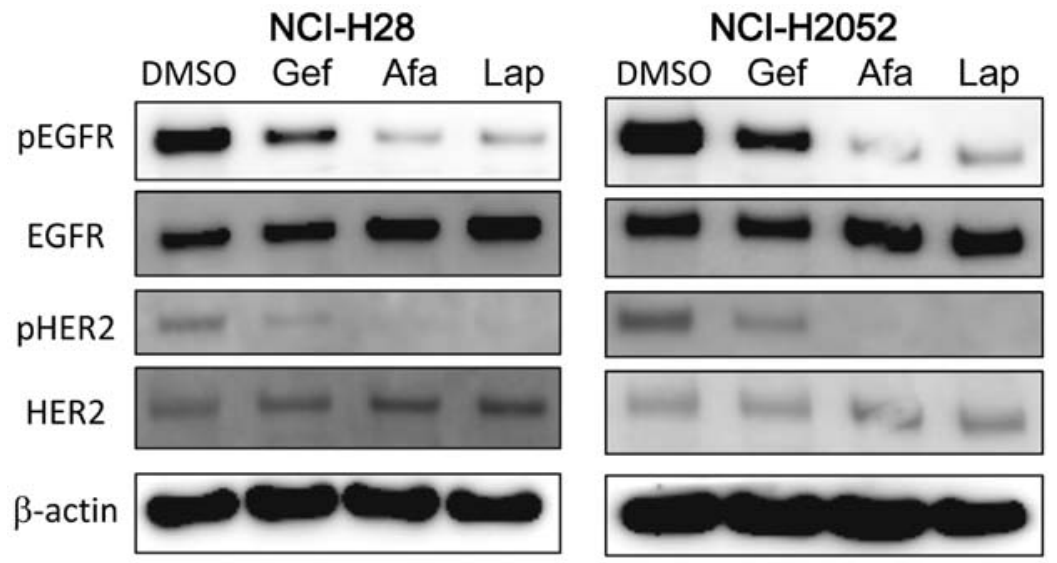

Figure 4. Effects of tyrosine kinase inhibitor on EGFR and HER2 signaling in malignant pleural mesothelioma cells. Western blot analysis showing total EGFR and HER2, as well as phosphorylated-EGFR (pEGFR) and -HER2 (pHER2) expression in cell lysates of both NCI-H28 and NCI-H2052 cells treated with each tyrosine kinase inhibitor (TKI) for $1 \mathrm{~h}$. DMSO treated cells served as control. Representative data of three independent experiments are shown.

A

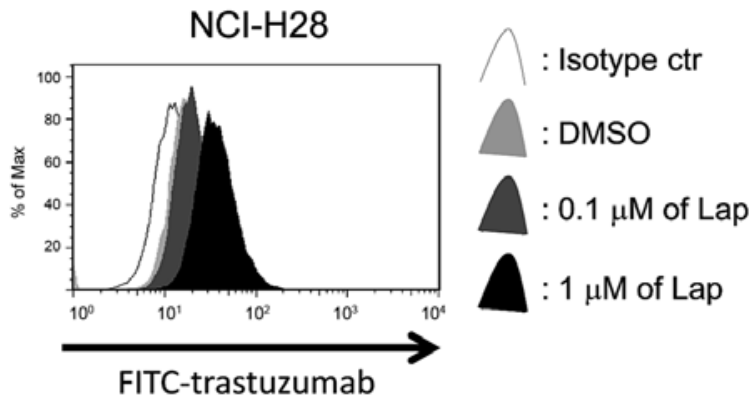

C

NCl-H28

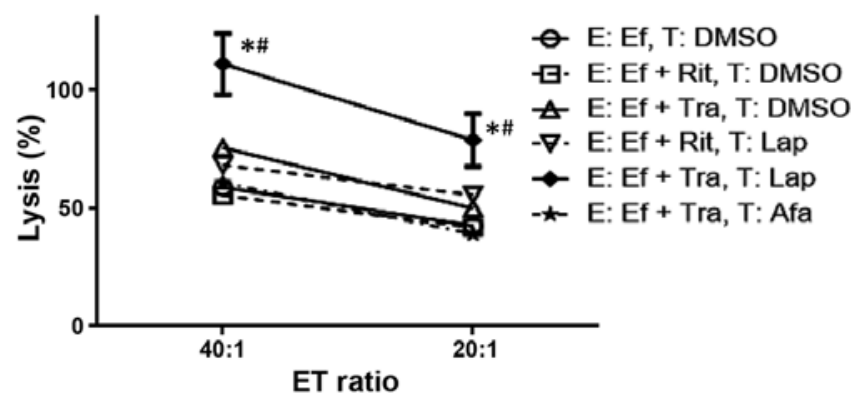

B

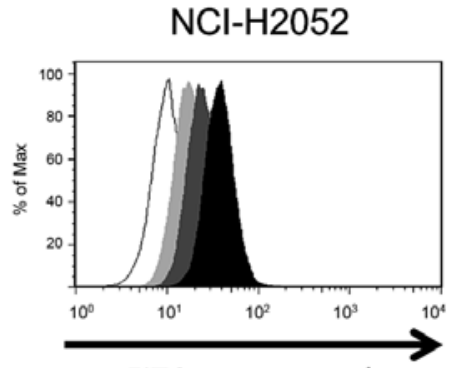

FITC-trastuzumab

D

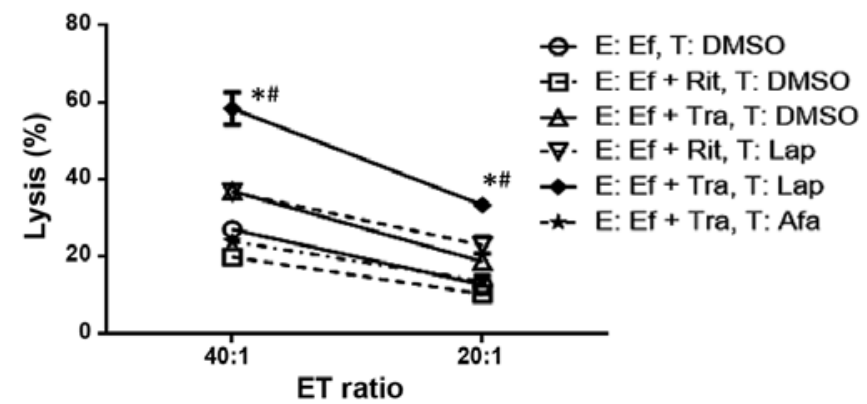

Figure 5. Lapatinib upregulated HER2 expression and enhanced trastuzumab-mediated antibody-dependent cellular cytotoxicity in malignant pleural mesothelioma cell lines. (A) NCI-H28 and (B) NCI-H2052 cells were treated with DMSO or $1 \mu \mathrm{M}$ of afatinib or lapatinib for $24 \mathrm{~h}$, then the binding site of trastuzumab was analyzed using FITC-conjugated trastuzumab. FITC-conjugate rituximab was used as an isotype control. Representative data of three independent experiments are shown. (C) NCI-H28 and (D) NCI-H2052 cells were treated with DMSO or $1 \mu \mathrm{M}$ of lapatinib or afatinib for $24 \mathrm{~h}$, then trastuzumab-mediated antibody-dependent cellular cytotoxicity (ADCC) was assessed using the LDH release assay. Representative data of three independent experiments (MPM cell lines) are shown. The values are presented as the mean \pm SD of triplicate samples. E:T ratio; effector/target ratio. ${ }^{*} \mathrm{p}<0.05$ vs. E: Ef + Tra, T: DMSO, ${ }^{\#}$ p $<0.01$ vs. E: Ef + Rit, T: Lap; Two-way ANOVA followed by Bonferroni's post test.

Lapatinib enhances trastuzumab or cetuximab to bind with MPM cells from a patient, resulted in enhanced cetuximab- or trastuzumab-mediated ADCC. Finally, MPM-p3 cells, which were collected from a malignant effusion of a patient with MPM, were assessed. Both EGFR and HER2 were upregulated by lapatinib in MPM-p3 cells (Fig. 3). In line with these results, lapatinib upregulated both the cetuximab and trastuzumab binding sites (Fig. 6A and B), resulting in enhanced cetuximab- or trastuzumab-mediated ADCC in MPM-p3 cells (Fig. 6C and D).

\section{Discussion}

In the present study, we reported that lapatinib enhanced trastuzumab-mediated ADCC in MPM cell lines as well as trastuzumab- and cetuximab-mediated ADCC in patient-derived MPM cells. Although EGFR is frequently expressed in MPM patient cells, EGFR targeting therapy using first-generation EGFR-TKIs gefitinib or erlotinib have failed to show any clinical benefits in previously conducted studies $(4,5)$. HER2 is also expressed in MPM cells, however, 
A

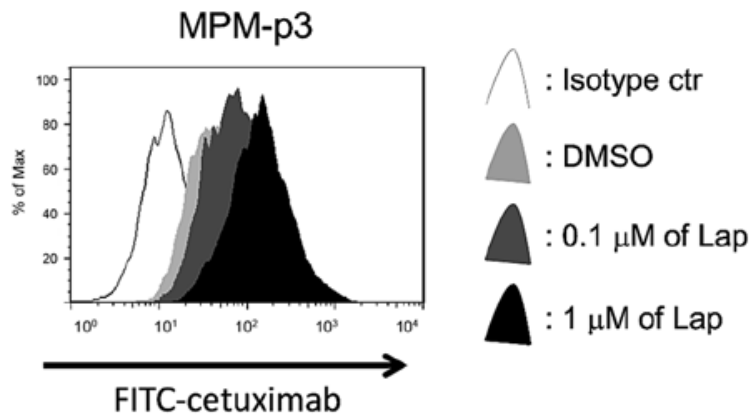

C MPM-p3 with cetuximab

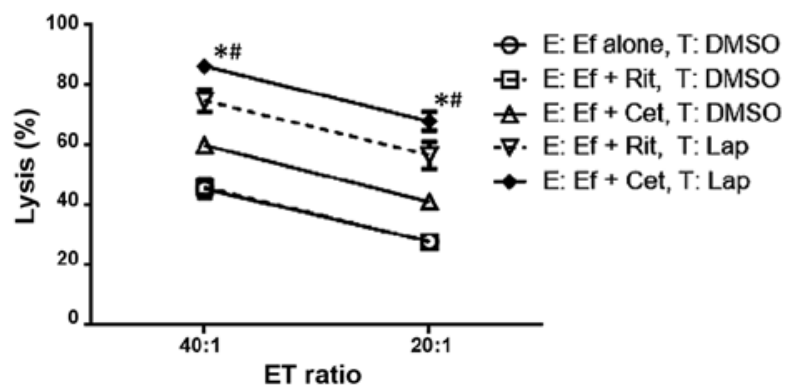

B

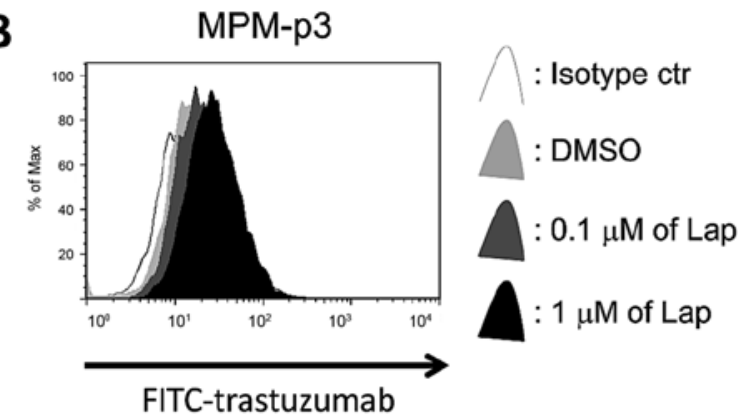

D

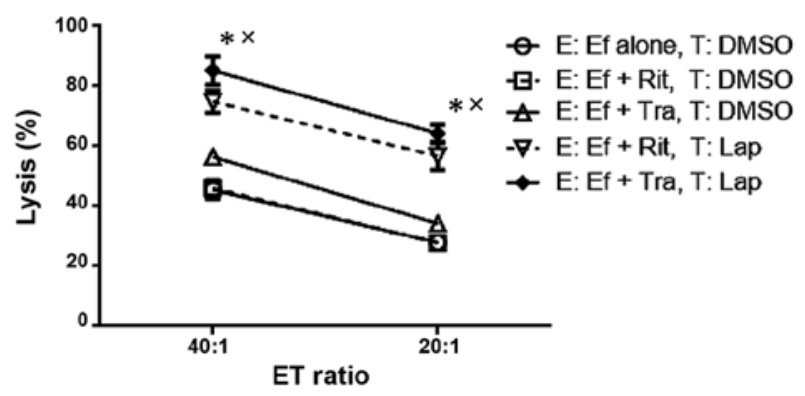

Figure 6. Lapatinib enhances cetuximab-/trastuzumab-mediated antibody-dependent cellular cytotoxicity via upregulations of EGFR and HER2 in patient-derived malignant pleural mesothelioma cells. Patient-derived MPM-p3 cells were treated with DMSO or $1 \mu \mathrm{M}$ of lapatinib for $24 \mathrm{~h}$, then the binding site of (A) cetuximab or (B) trastuzumab were analyzed using FITC-conjugated trastuzumab. FITC-conjugate rituximab was used as an isotype control. Single experiments are shown. Patient-derived MPM-p3 cells were treated with DMSO or $1 \mu \mathrm{M}$ of lapatinib for $24 \mathrm{~h}$, both cetuximab-mediated (C) and trastuzumabmediated (D), antibody-dependent cellular cytotoxicity (ADCC) was assessed. Single experiments are shown. E:T ratio, effector/target ratio. ${ }^{*}$ p<0.05 vs. E: Ef + Rit, T: Lap, ${ }^{\#}$ p<0.05 vs. E: Ef + Cet, T: DMSO, ${ }^{\times}$p<0.05 vs. E: Ef + Tra, T: DMSO; Two-way ANOVA followed by Bonferroni's post test.

there are no reported clinical studies using trastuzumab or lapatinib. Thus, targeting both EGFR and HER2 in patients with MPM is still an undeveloped yet promising strategy. Other EGFR- or HER2-targeting regents are antibody drugs targeting EGFR or HER2. The main mechanism behind the therapeutic potential of the anti-HER 2 antibody drug trastuzumab in HER2 overexpressing breast cancer cells is ADCC (14), which is also a crucial mechanism for the antitumor effects of anti-EGFR antibody drug cetuximab in both NSCLC (15) and MPM cells (16). For antibody drugs, not the driver mutation, but the overexpression of the targeting molecule is important for clinical benefit, suggesting that the upregulation of the expression of EGFR or HER2 by TKI is a reasonable strategy.

We have shown that MPM cells frequently expressed both EGFR and HER2 molecules, but did not express HER3, suggesting that both EGFR and HER2 could be promising targets for the treatment of MPM. We have also shown that dual EGFR/HER2-TKI lapatinib enhanced the expression of EGFR or HER2, however, the EGFR-TKI gefitinib and pan-HER TKI afatinib did not enhance either receptor in MPM cells.

Although these results are in line with the recent studies showing that lapatinib enhanced trastuzumab-mediated ADCC in breast cancer or gastrointestinal cancers (6-8), we believe that the present study is promising as there is no established second-line therapy for patients with MPM, and new treatment strategies are urgently required.

Another important finding is that dual EGFR/HER2-TKI lapatinib enhances both EGFR and HER2, while neither the
EGFR-TKI gefitinib nor pan-HER TKI afatinib have shown this effect. Our immunoblotting results show the phosphorylation of both EGFR and HER2 were weakly inhibited by gefitinib, while strongly inhibited by afatinib or lapatinib, suggesting that gefinitib had weaker effects on EGFR/HER2 signaling than afatinib or lapatinib in MPM cells. Notably, afatinib shows a similar effect to that of lapatinib on both pEGFR and pHER2, but differed from that of lapatinib on the expression of HER2. Rimawi et al showed that afatinib monotherapy decreased the HER2 dimer in breast cancer tissue collected from patients (17). It was also reported that lapatinib blocks the internalization of HER2, resulting in enhanced stabilization of inactive HER2 homo- and heterodimers in the plasma membrane of breast cancer cells (6). Based on these reported findings, the possible mechanism is that lapatinib keeps homo- or heterodimers on the cell surface via the inhibition of HER2 internalization, resulting in the enhancement of HER2 expression. However, if afatinib does not keep the dimerization, then HER2 may be internalized and degradated.

It is well known that the first-generation EGFR-TKIs gefitinib and erlotinib exhibit a significant response in NSCLC cells having the EGFR driver mutation. However, this mutation is quite rare in MPM cells; therefore, first-generation EGFR-TKIs have no clinical benefits for patients with MPM. In contrast, the second-generation EGFR-TKI afatinib can inhibit EGFR signaling in NSCLC cells without EGFR driver mutation or with both EGFR driver mutation and a resistant mutation such as the T790M mutation. According to our data, both afatinib and lapatinib clearly inhibited the cell 
proliferation of MPM cells, suggesting that these TKIs may show the same clinical benefits as monotherapy. Additionally, we have shown in the present study that lapatinib enhanced trastuzumab- or cetuximab-mediated ADCC while afatinib did not. This suggests that lapatinib combined with trastuzumab and/or cetuximab is a very promising strategy, as we can expect the dual EGFR/HER2 blocking effect of lapatinib, as well as trastuzumab- or cetuximab-mediated ADCC with this combination. We should pursue optimal effect with least toxicity for MPM patients, and lapatinib has been shown to be an ideal partner drug for cetuximab or trastuzumab to enhance ADCC for MPM treatment.

\section{Acknowledgements}

We thank Ms. Maitani and the staff of the Tissue Culture and Immunology, Biochemistry and Tissue Biology and Electron Microscopy Research Center (Kawasaki Medical School) for technical assistance. We also thank Editage for English language editing. This study was supported by JSPS Kakenhi Grant no. 25462189, Kawasaki Medical School Project Grant (25-34), the Ryobi Teien Research Grant and The Okayama Medical Foundation (to R.O.), and the Strategic Research Foundation Grant-aided Project for Private Universities from Ministry of Education, Culture, Sport, Science and Technology (to M.N.).

\section{References}

1. Rusch VW and Venkatraman ES: Important prognostic factors in patients with malignant pleural mesothelioma, managed surgically. Ann Thorac Surg 68: 1799-1804, 1999.

2. Vogelzang NJ, Rusthoven JJ, Symanowski J, Denham C, Kaukel E, Ruffie P, Gatzemeier U, Boyer M, Emri S, Manegold C, et al: Phase III study of pemetrexed in combination with cisplatin versus cisplatin alone in patients with malignant pleural mesothelioma. J Clin Oncol 21: 2636-2644, 2003.

3. Destro A, Ceresoli GL, Falleni M, Zucali PA, Morenghi E, Bianchi P, Pellegrini C, Cordani N, Vaira V, Alloisio M, et al: EGFR overexpression in malignant pleural mesothelioma. An immunohistochemical and molecular study with clinico-pathological correlations. Lung Cancer 51: 207-215, 2006.

4. Garland LL, Rankin C, Gandara DR, Rivkin SE, Scott KM, Nagle RB, Klein-Szanto AJ, Testa JR, Altomare DA and Borden EC: Phase II study of erlotinib in patients with malignant pleural mesothelioma: A Southwest Oncology Group Study. J Clin Oncol 25: 2406-2413, 2007.

5. Govindan R, Kratzke RA, Herndon JE II, Niehans GA, Vollmer R, Watson D, Green MR and Kindler HL; Cancer and Leukemia Group B (CALGB 30101): Gefitinib in patients with malignant mesothelioma: A phase II study by the Cancer and Leukemia Group B. Clin Cancer Res 11: 2300-2304, 2005.
6. Scaltriti M, Verma C, Guzman M, Jimenez J, Parra JL, Pedersen K, Smith DJ, Landolfi S, Ramon y Cajal S, Arribas J, et al: Lapatinib, a HER2 tyrosine kinase inhibitor, induces stabilization and accumulation of HER 2 and potentiates trastuzumab-dependent cell cytotoxicity. Oncogene 28: 803-814, 2009.

7. Mimura K, Kono K, Maruyama T, Watanabe M, Izawa S, Shiba S, Mizukami Y, Kawaguchi Y, Inoue M, Kono T, et al: Lapatinib inhibits receptor phosphorylation and cell growth and enhances antibody-dependent cellular cytotoxicity of EGFRand HER2-overexpressing esophageal cancer cell lines. Int J Cancer 129: 2408-2416, 2011.

8. Shiraishi K, Mimura K, Izawa S, Inoue A, Shiba S, Maruyama T, Watanabe M, Kawaguchi Y, Inoue M, Fujii H, et al: Lapatinib acts on gastric cancer through both antiproliferative function and augmentation of trastuzumab-mediated antibody-dependent cellular cytotoxicity. Gastric Cancer 16: 571-580, 2013.

9. Usami N, Fukui T, Kondo M, Taniguchi T, Yokoyama T, Mori S, Yokoi K, Horio Y, Shimokata K, Sekido Y, et al: Establishment and characterization of four malignant pleural mesothelioma cell lines from Japanese patients. Cancer Sci 97: 387-394, 2006.

10. Pao W, Miller VA, Politi KA, Riely GJ, Somwar R, Zakowski MF, Kris MG and Varmus H: Acquired resistance of lung adenocarcinomas to gefitinib or erlotinib is associated with a second mutation in the EGFR kinase domain. PLoS Med 2: e73, 2005.

11. Li D, Ambrogio L, Shimamura T, Kubo S, Takahashi M, Chirieac LR, Padera RF, Shapiro GI, Baum A, Himmelsbach F, et al: BIBW2992, an irreversible EGFR/HER2 inhibitor highly effective in preclinical lung cancer models. Oncogene 27: 4702-4711, 2008

12. Geyer CE, Forster J, Lindquist D, Chan S, Romieu CG, Pienkowski T, Jagiello-Gruszfeld A, Crown J, Chan A, Kaufman B, et al: Lapatinib plus capecitabine for HER2-positive advanced breast cancer. N Engl J Med 355: 2733-2743, 2006.

13. Gilmer TM, Cable L, Alligood K, Rusnak D, Spehar G, Gallagher KT, Woldu E, Carter HL, Truesdale AT, Shewchuk L, et al: Impact of common epidermal growth factor receptor and HER 2 variants on receptor activity and inhibition by lapatinib. Cancer Res 68: 571-579, 2008.

14. Clynes RA, Towers TL, Presta LG and Ravetch JV: Inhibitory Fc receptors modulate in vivo cytotoxicity against tumor targets. Nat Med 6: 443-446, 2000.

15. Kurai J, Chikumi H, Hashimoto K, Yamaguchi K, Yamasaki A, Sako T, Touge H, Makino H, Takata M, Miyata M, et al: Antibody-dependent cellular cytotoxicity mediated by cetuximab against lung cancer cell lines. Clin Cancer Res 13: 1552-1561, 2007.

16. Kurai J, Chikumi H, Hashimoto K, Takata M, Sako T, Yamaguchi K, Kinoshita N, Watanabe M, Touge H, Makino H, et al: Therapeutic antitumor efficacy of anti-epidermal growth factor receptor antibody, cetuximab, against malignant pleural mesothelioma. Int J Oncol 41: 1610-1618, 2012.

17. Rimawi MF, Aleixo SB, Rozas AA, Nunes de Matos Neto J, Caleffi M, Figueira AC, Souza SC, Reiriz AB, Gutierrez C, Arantes H, et al: A neoadjuvant, randomized, open-label phase II trial of afatinib versus trastuzumab versus lapatinib in patients with locally advanced HER2-positive breast cancer. Clin Breast Cancer 15: 101-109, 2015. 